Supplementary Information for:

\title{
Adsorption and Motion of Single Molecular Motors on $\mathrm{TiO}_{2}(110)$
}

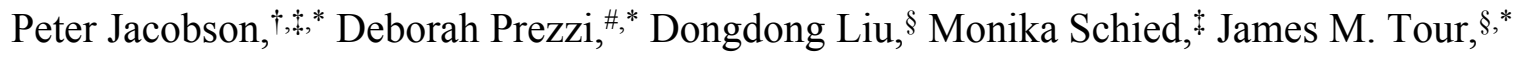

Stefano Corni, ${ }^{\#, \&}$ Arrigo Calzolari, ${ }^{\#}$ Elisa Molinari,,,$\epsilon$ and Leonhard Grill ${ }^{\ddagger}, *$

†School of Mathematics and Physics, The University of Queensland, QLD 4072, Brisbane, Australia

Department of Physical Chemistry, University of Graz, Heinrichstrasse 28, 8010 Graz, Austria \#Nanoscience Institute of CNR, S3 Center, 41125 Modena, Italy

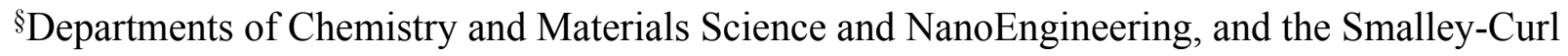
Institute and NanoCarbon Center, Rice University, Houston, Texas 77005, USA

\&Dipartimento di Scienze Chimiche, Università di Padova, I-35131 Padova, Italy

${ }^{\epsilon}$ Department of Physics, Mathematics, and Informatics, University of Modena and Reggio Emilia, 41121 Modena, Italy

Email: p.jacobson@uq.edu.au,deborah.prezzi@nano.cnr.it, tour@rice.edu, leonhard.grill@unigraz.at 


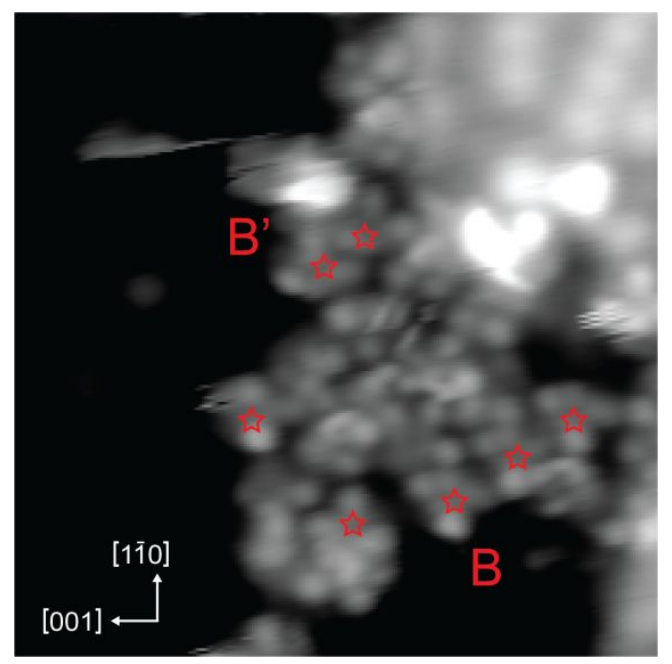

Supplementary Figure 1: Disordered molecular island nucleated at a lower step edge. This island contains conformation B, B', and undetermined conformations. Red stars indicate B/B' molecules. 


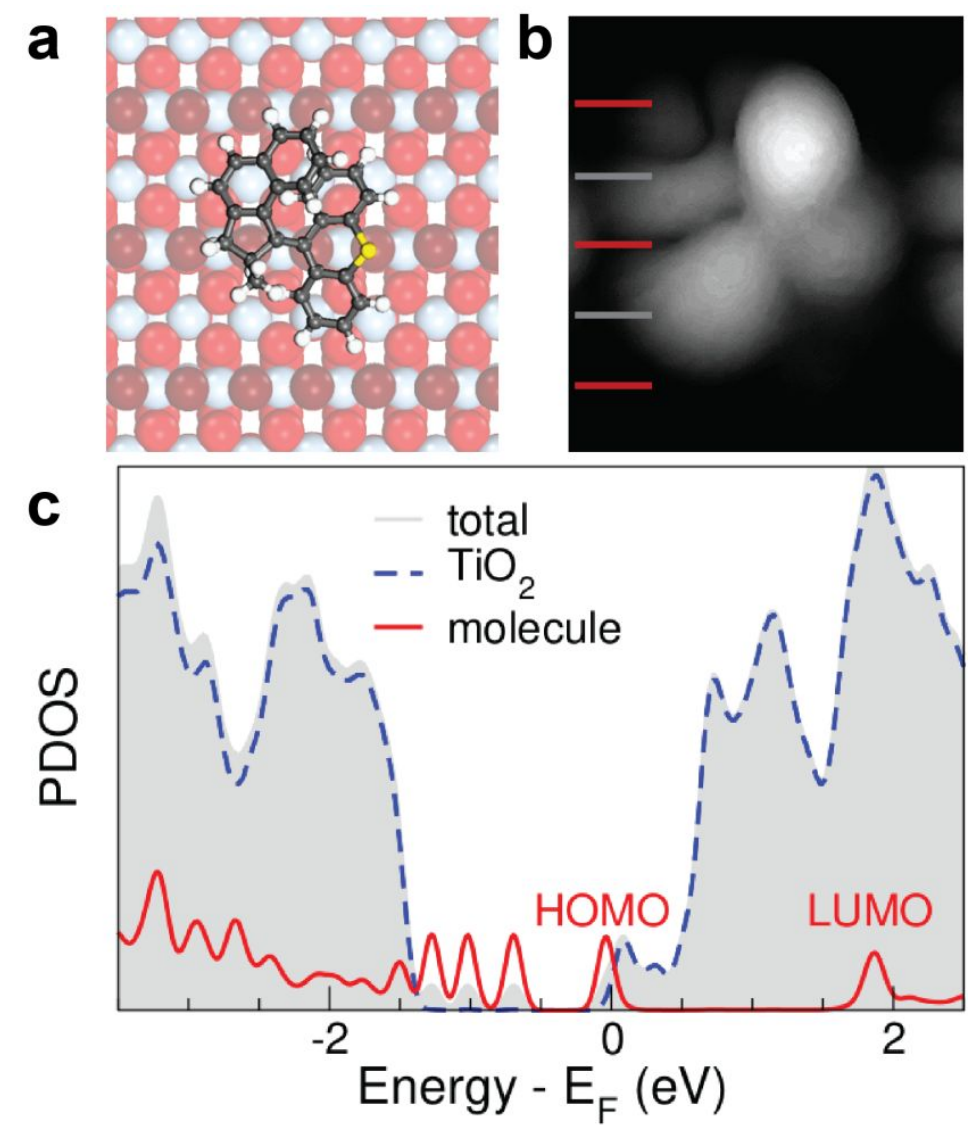

Supplementary Figure 2: a) Top view of the adsorption configuration of conformation A in the absence of surface hydroxyls and polaronic effects. b) Simulated STM images with the $\mathrm{O}_{2 \mathrm{c}}$ (red) and $\mathrm{Ti}_{5 \mathrm{c}}$ (grey) rows indicated. c) Partial density of states for the molecule and surface. HOMO denotes the highest occupied molecular orbital, LUMO the lowest unoccupied molecular orbital. 

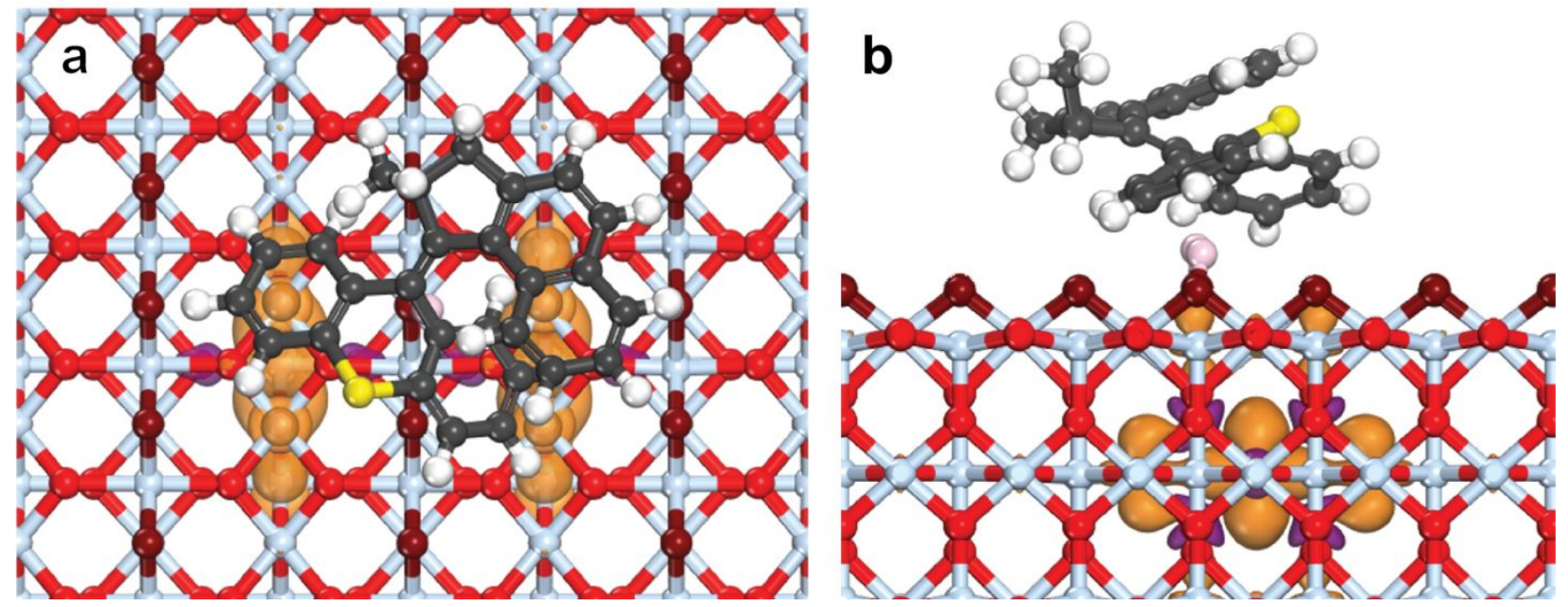

Supplementary Figure 3: a) Top-view of the spin polarization density due to the polaronic

triplet state, corresponding side-view (presented in Figure 4) in panel b.
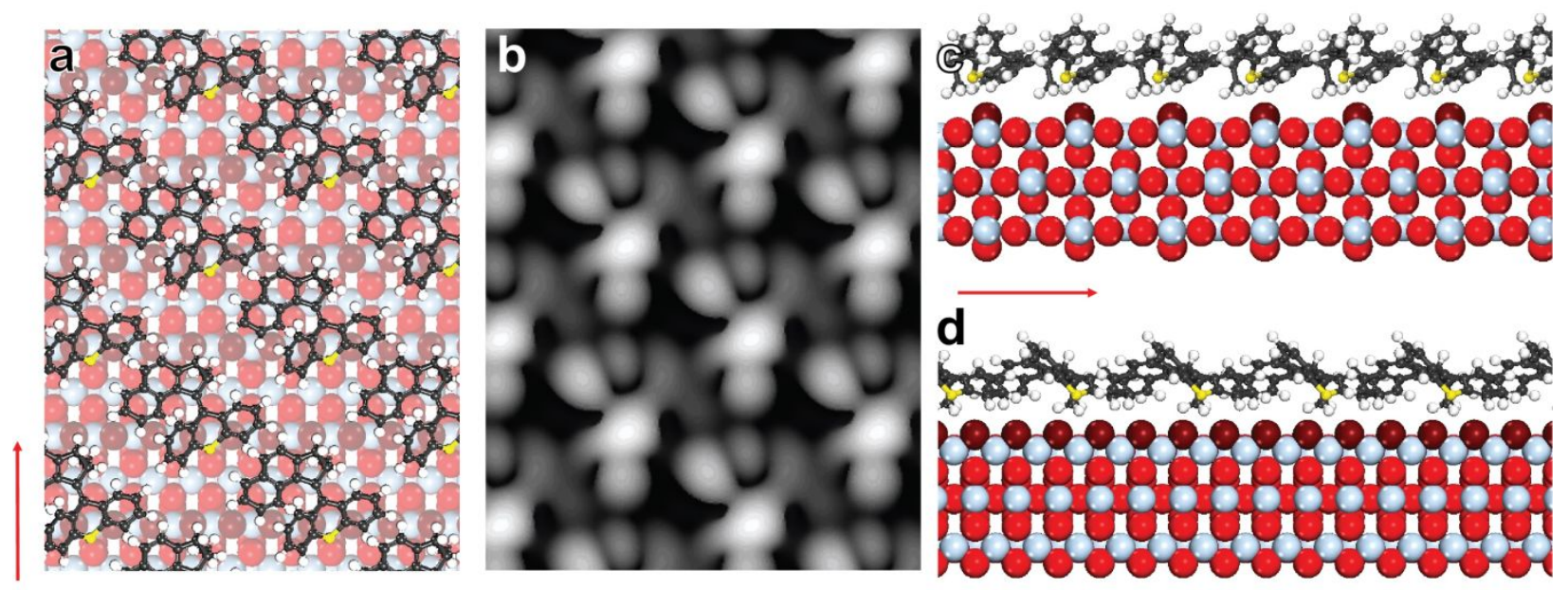

Supplementary Figure 4: a) Top-view of relaxed molecular layer structure on $\operatorname{TiO}_{2}(110)$. b)

Simulated STM image with the same scale as panel a (presented in Figure 5). c, d) Side-views of the molecular overlayer. 


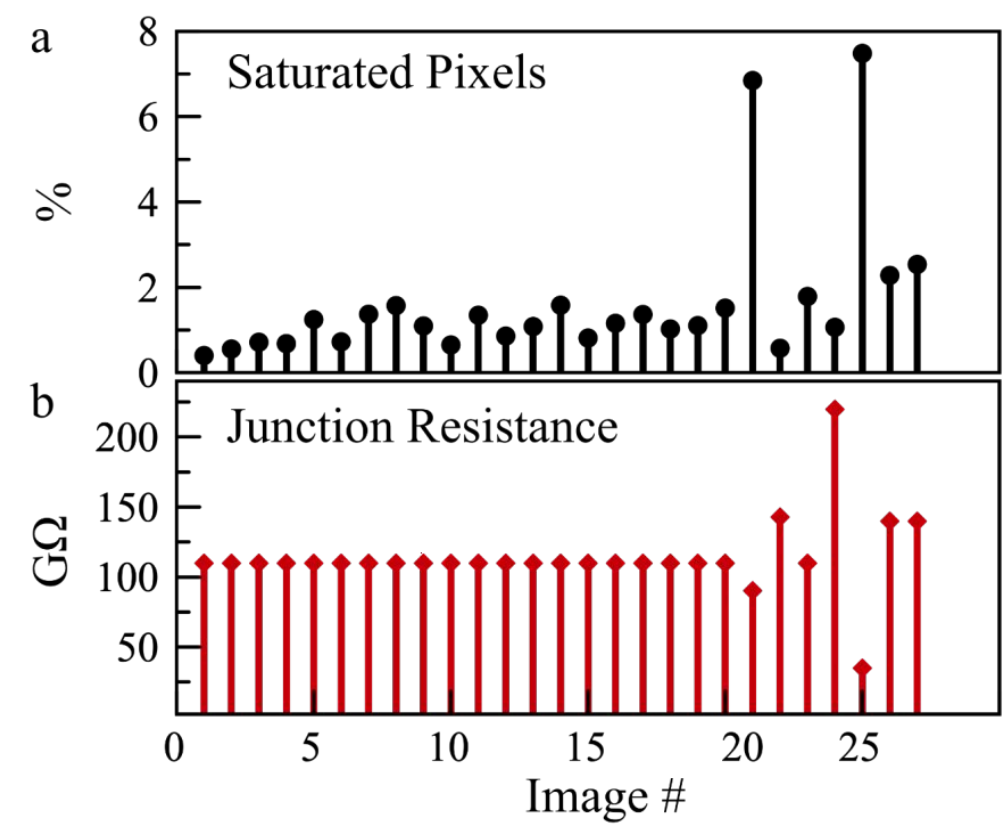

Supplementary Figure 5: a) Scratch analysis for Supplementary Movie 2 and tunnel junction resistance (b) for each frame of the movie. 


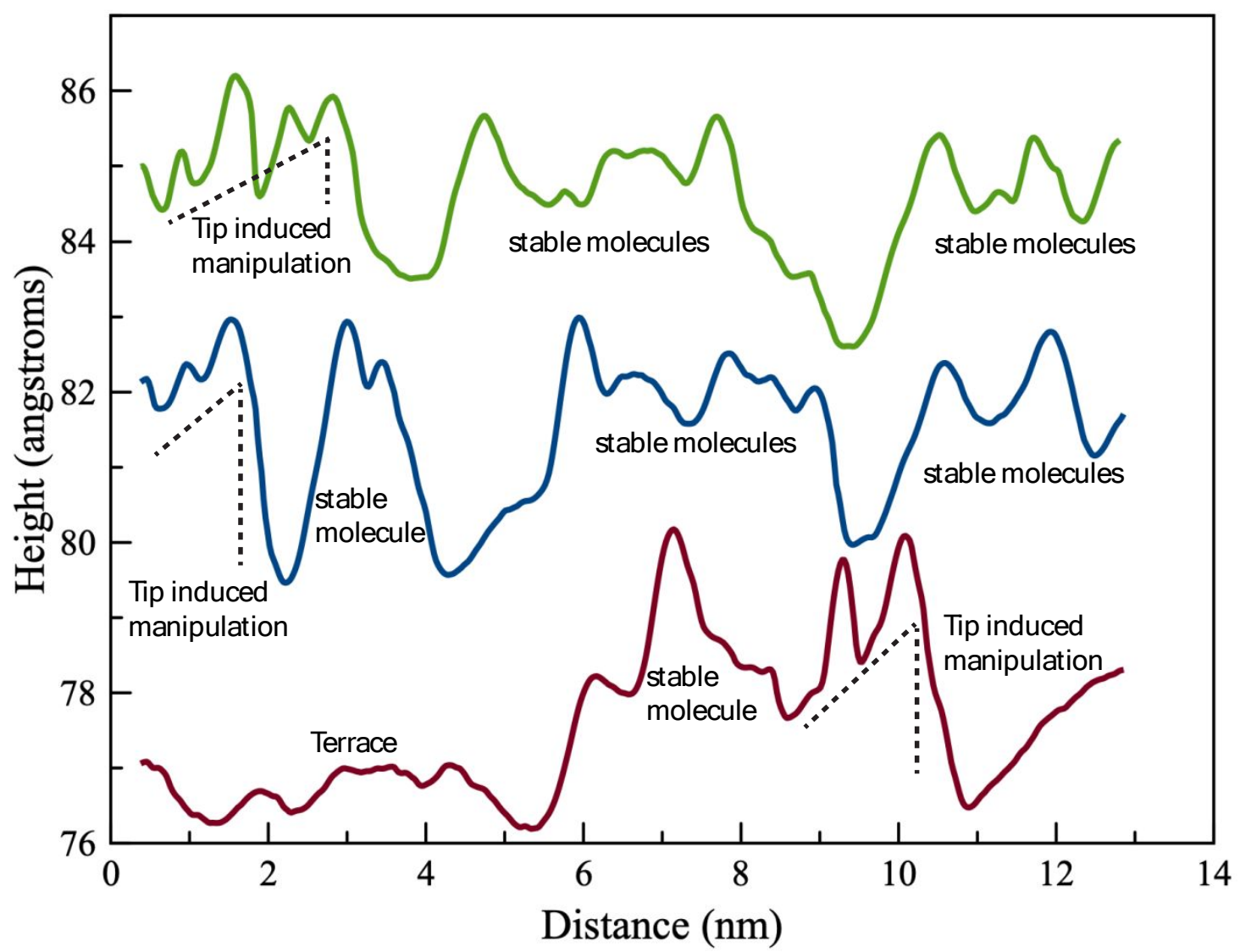

Supplementary Figure 6: Line profiles along the fast scanning direction (scanning left to right;

$12^{\circ}$ off the [001] direction) showing characteristic tip induced manipulation traces from

Supplementary Movie 1. Tunneling parameters for all line profiles: $+1.54 \mathrm{~V}, 14 \mathrm{pA}$. 